
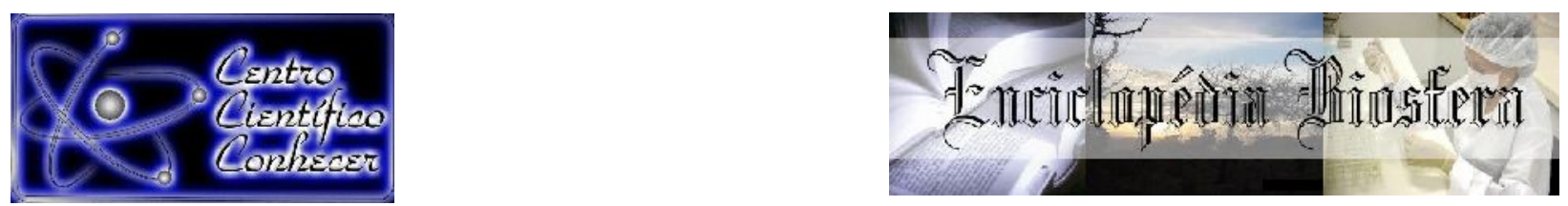

\title{
ANÁLISE HISTÓRICA SOBRE A HEMATOLOGIA EM PEIXES NO BRASIL: ESTUDO QUALI/QUANTITATIVO
}

\author{
Maria de los Angeles Perez Lizama ${ }^{1}$, Gilsemara dos Santos Cagni ${ }^{2}$, Felipe Zavaski ${ }^{3}$ \\ ${ }^{1}$ Professora Doutora do Curso de Ciências Biológicas e Medicina Veterinária e do \\ Mestrado em Tecnologias Limpas da Universidade Cesumar/ UNICESUMAR, \\ Maringá, Paraná, Brasil (maria.lizama@unicesumar.edu. ). Bolsista \\ ICETI/Unicesumar br \\ ${ }^{2}$ Engenheira Ambiental, aluna de doutorado em Biotecnologia Ambiental, \\ Universidade Estadual de Maringá, Paraná, Brasil (gscagni@hotmail.com) \\ ${ }^{3}$ Biólogo, aluno do mestrado em Tecnologias Limpas da Univeridade Cesumar/ \\ UNICESUMAR, Maringá, Paraná, Brasil. Bolsista Unicesumar
}

Recebido em: 15/11/2020 - Aprovado em: 15/12/2020 - Publicado em: 30/12/2020 DOI: 10.18677/EnciBio_2020D20

\begin{abstract}
RESUMO
A diversidade biológica é frequentemente empregada como indicador da saúde ambiental, sendo que qualquer alteração que ocorra no hábitat é refletida em variações das populações presentes no ecossistema. Alguns padrões sanguíneos de vidas aquáticas podem ir além da delimitação dos valores de referências para espécies, podendo ser usada como instrumento auxiliar rápido no diagnóstico de doenças. A exposição dos peixes em ambientes poluídos causa infinitas alterações moleculares, estas alterações podem ser analisadas no eritrograma, leucograma e íons plasmáticos e são bem eficientes, apontando correlações direta com a presença de poluentes. O objetivo deste trabalho foi realizar um estudo quali/quantitativo do perfil hematológico em peixes no Brasil, verificar a importância destes parâmetros para os estudos de monitoramento ambiental, bem para a sanidade destes animais. A partir de 1997, estudos sobre hematologia em peixes foram abordados por diversos motivos, principalmente com relação ao conhecimento dos índices hematológicos em peixes. A partir desta data, estes estudos apresentaram incremento, havendo uma grande preocupação com dietas mais balanceadas que aumentam a produtividade e a sanidade dos animais. Os trabalhos se referem tanto a animais em confinamento como de ambiente natural, porém, as espécies mais estudadas são as de água doce, devido a que nas espécies marinhas, a produção é bem menor. Os parâmetros hematológicos são utilizados como biomarcadores da saúde animal mas, também no biomonitoramento ambiental, uma importante ferramenta, pois é possível verificar as alterações nos animais sem haver a necessidade de sacrificar o animal.
\end{abstract}

PALAVRAS-CHAVE: Hematologia de peixes; ictioparasitologia; monitoramento ambiental. 


\title{
HEMATOLOGY IN FISH IN BRAZIL: QUALI / QUANTITATIVE STUDY OF NATIONAL PUBLICATIONS
}

\begin{abstract}
Biological diversity is often used as an indicator of environmental health, and any alteration that occurs in the habitat is reflected in variations of the populations present in the ecosystem. Some blood patterns of aquatic lives may go beyond the delimitation of reference values for species, and can be used as a rapid auxiliary tool in the diagnosis of diseases. The exposure of fish in polluted environments causes infinite molecular changes, these changes can be analyzed in erythrogram, leukogram and plasma ions are very efficient, indicating direct correlations with the presence of pollutants. The objective of this work was to conduct a qualitative/quantitative study of the hematological profile in fish in Brazil,to verify the importanceof these parameters for environmental monitoring studies, as well as for the health of these animals. Since 1997, studies on hematology in fish have been addressed for several reasons, mainly regarding the knowledge of hematological indices in fish. From this date, these studies showed ana aument increase, withone ofgreat concernswith more balanced dietas thatincreases the productivity and health of the animals.. The studies refer to both animals in confinement and the natural environment, however, the most studied species are freshwater, due to which in marine species, the production is much lower. Hematological parameters are used as biomarkers of animal health but also in environmental biomonitoring,an importanttool, because it is possible to verify changes in animals without the need to sacrifice the animal.
\end{abstract}

KEYWORDS: Fish hematology; ichthyoparasitology; environmental monitoring.

\section{INTRODUÇÃO}

A diversidade de organismos que habitam 0 ambiente aquático é extremamente variada. Dentre esses organismos os peixes representam aproximadamente $50 \%$ das espécies de vertebrados, englobando cerca de 32.000 espécies que ocupam os mais diversos ambientes aquáticos. Os peixes possuem grande importância comercial, considerando que fazem parte da dieta humana em muitos países. Por outro lado, em algumas regiões, são considerados a principal fonte de proteínas da população (MARTINEZ; COLU, 2002).

$\mathrm{O}$ fato de serem importantes comercialmente não necessariamente os colocam como bons indicadores ambientais. Ainda assim é crescente o número de estudos nos quais os peixes são utilizados como bioindicadores da qualidade dos ecossistemas aquáticos (MARTINEZ; COLU, 2002; LIZAMA et al., 2013).

A intensificação nos sistemas de produção de organismos aquáticos, com o intuito de aumento na produtividade e crescimento da atividade aquícola brasileira, mostrou vários obstáculos, sendo que um é a sanidade destes organismos. Os danos causados aos peixes estão relacionados, entre outros fatores, a dieta e suplementação, ao parasitismo, o uso excessivo de medicamentos está relacionado à sanidade e ao bem-estar do hospedeiro, e também ao ambiente (CONTE, 2004; TACHIBANA et al., 2019).

As exposições desses organismos aquáticos a poluentes provocam diferentes alterações bioquímicas, moleculares e fisiológicas, que podem ser empregadas como importantes ferramentas a fim de analisar os riscos ambientais. Neste contexto, análises hematológicas em peixes submetidos aos contaminantes podem 
ser consideradas emergentes no sentido de monitorar a qualidade ambiental (SERIANI; RANZANI-PAIVA, 2012).

A exposição a pesticidas e efluentes industriais e orgânicos, além de metais pesados, muitas vezes dificultam o transporte do oxigênio e acarretam hipóxia funcional. Tais deformações levam a um estresse fisiológico, podendo promover danos instantâneos ou graduais aos peixes (SERIANI; RANZANI-PAIVA, 2012).

Segundo Tavares-Dias et al. (2009) os parâmetros bioquímicos metabólicos e hematológicos podem fornecer informações relevantes sobre as condições fisiológicas e a saúde do espécime, bem como de sua população. Entretanto são imprescindíveis as informações relativas às propriedades do sangue para 0 conhecimento desses parâmetros.

A hematologia é o estudo do sangue ou a soma de conhecimentos sobre o mesmo, a determinação de valores dos parâmetros hematológicos em condições normais e anormais. Porém, para que sejam utilizados parâmetros hematológicos de peixes como indicadores do estado fisiológico é necessário que existam padrões para poder compará-los, uma vez que distintos manejos, habitats, hábitos e alimentações podem influenciar nos valores finais desses parâmetros.

O objetivo deste trabalho foi realizar uma abordagem analítica histórica, quali e quantitativa da literatura científica, sobre os estudos com os parâmetros hematológicos como ferramentas de bem estar animal, bem como no biomonitoramento ambiental.

\section{MATERIAIS E MÉTODOS}

Foi realizado um estudo quali/quantitativo acerca dos trabalhos com hematologia de peixes a partir do ano de 1997. Foram utilizados como base artigos científicos completos publicados em jornais, livros e encontrados na plataforma CAPES, base SciELO e Web of Science.

Os dados foram unificados por meio de uma avaliação Bibliográfica de estudos que correlacionam a hematologia de peixes no Brasil. A pesquisa foi conduzida usando as bases on line: Isi Web of Knowledge (https://webofknowledge.com), Scientific Electronic Library Online - Scielo (http: /www.scielo.org/php/index.php), bem como o site do Google Scholar (https://scholar.google.com.br/), em português e inglês, utilizando os descritores "Hematologia de peixes no Brasil", "Hematol*", "Hematology", "peixes", "fish", "biology", , "Hematologia" e "peixe". Realizando portando uma padronização e compilando os principais periódicos a partir de materiais eletrônicos.

A base de dados foi composta de artigos completos revisados por pares, a partir de revisões de periódicos, indexados ou não na base de dados utilizadas neste estudo. Os dados incluíram peixes de água doce e marinhos, tanto de ambientes naturais, pisciculturas e também, experimentos.

A compilação dos dados para os tópicos utilizados ocorreu de julho a novembro 2020. Após revisão do título, palavras-chave e resumo, foram selecionados um total de 131 estudos, sendo que destes por meio de avaliação minuciosa dos artigos, os mesmos obedeceram o seguinte critério de exclusão: repetição de artigos, ou sem aderência ao tema abordado. Dos 131 artigos, 23 foram excluidos, totalizando 108 artigos científicos dentro da plataforma. 


\section{RESULTADOS E DISCUSSÃO}

Dos 108 artigos válidos para este trabalho, a frequência com que estes artigos científicos foram publicados por ano, pode ser observada na Figura 1. Os estudos sobre hematologia de peixes no Brasil se iniciaram na década de 90, com a avaliação dos parâmetros hematológicos em Oreochromis niloticus (UEDA et al., 1997). A partir de 2000, foram iniciados estudos acerca dos parâmetros hematológicos de espécies de importância comercial como Schizodon borellii e Prochilodus lineatus na planície de inundação do alto rio Paraná, juntamente com outras espécies de elevado valor comercial (TAKEMOTO et al., 2000; EIRAS et al., 2000).

A partir de 2008, houve um crescente aumento dos estudos acerca dos padrões hematológicos em peixes no Brasil, sendo que nos anos de 2011 e 2012, a frequência tornou-se mais elevada em decorrência de inúmeros estudos sobre a influência da dieta alimentar em animais em cativeiro, auxiliando desta forma, a aumentar a produção. Apesar de ter dados incompletos para o ano de 2020 é possível verificar um aumento das frequências de estudos publicados em revistas científicas de renome.

FIGURA 1. Frequência de artigos acerca de hematologia de peixes publicados entre 1997 e 2020.

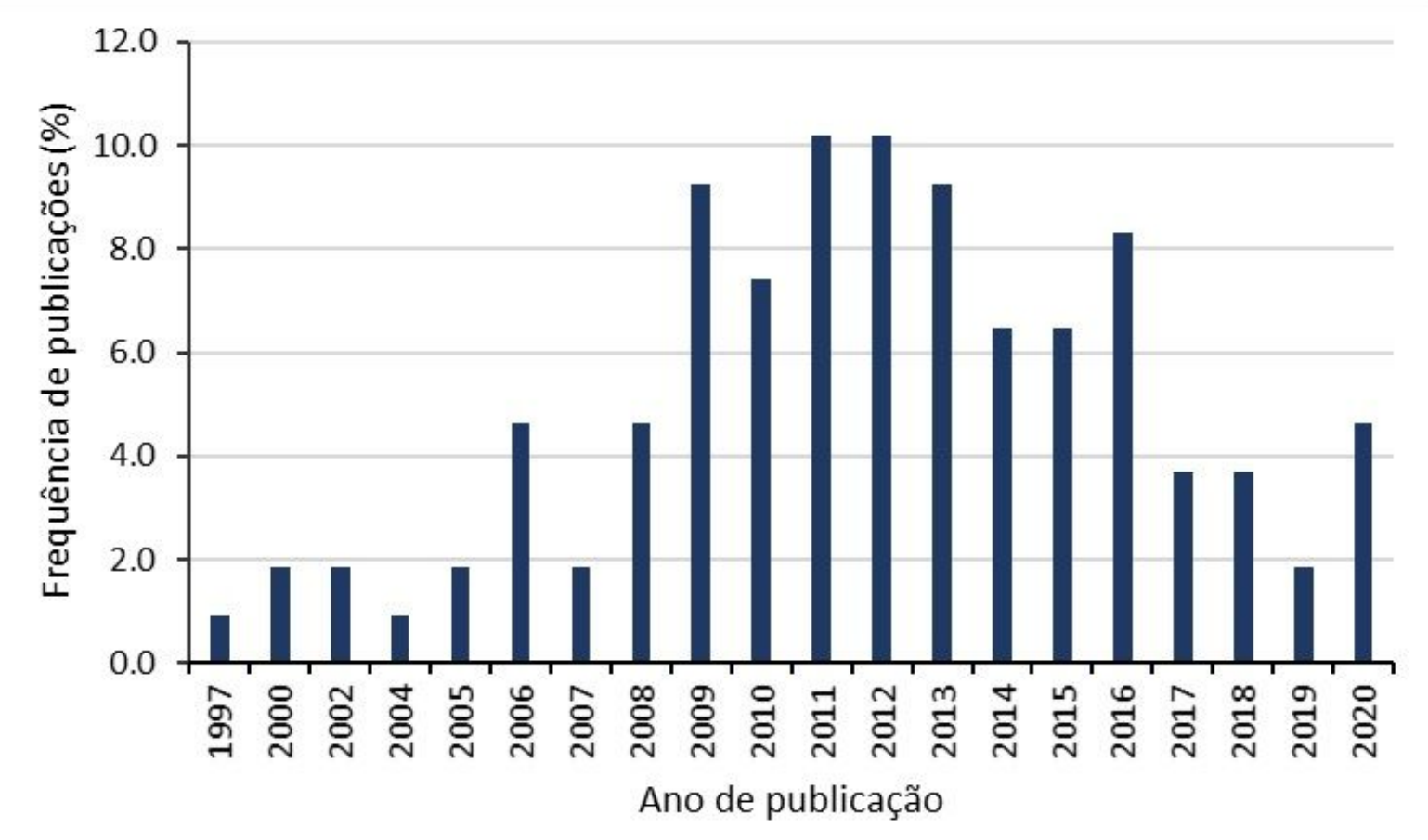

Fonte: Os autores (2020).

A tilápia, Oreochromis niloticus, é a espécie mais estudada (Figura 2). Isto se deve ao fato de que esta espécie é muito produzida no Brasil. De acordo com a Associação Brasileira da Piscicultura (2020) o Brasil produziu em 2019, 758.006 toneladas de tilápia para consumo interno e para exportação, contabilizando $57 \%$ da produção interna, elevando o Brasil a $4^{\circ}$ produtor mundial deste pescado. A aceitação pela rusticidade e palatabilidade da espécie faz com que estudos sobre a sua saúde e dieta sejam cada vez mais elevados, como observado em Schalch et al. (2015). 
FIGURA 2. Espécies de peixes registradas nos estudos científicos sobre os parâmetros hematológicos.

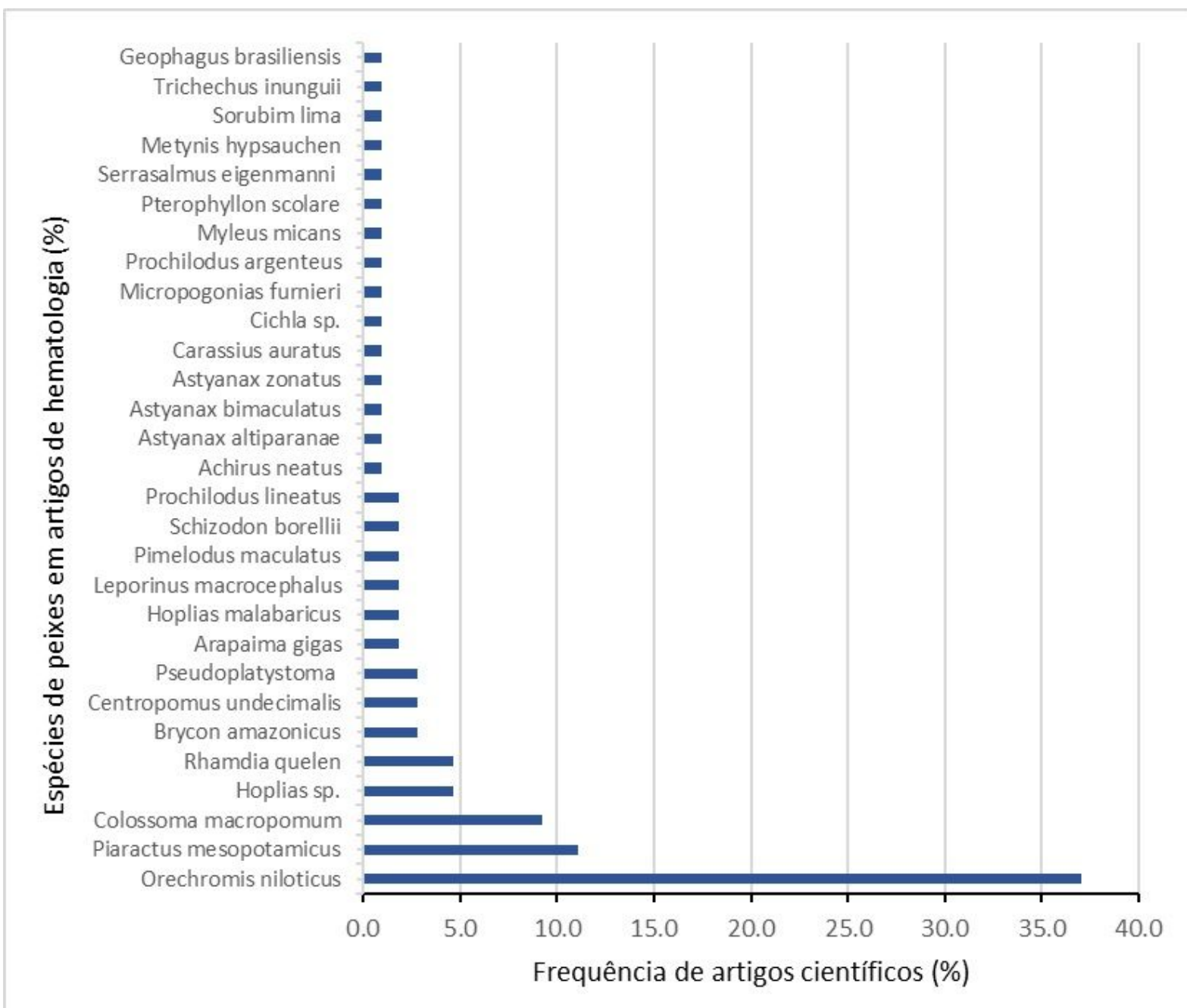

Fonte: Os autores (2020).

Entre as espécies nativas, o pacú (Piaractus mesopotamicus) e o tambaqui (Colossoma macropomum) também apresentam elevada frequência, pois estas duas espécies e os híbridos como a patinga e o tambacu são muito utilizados na produção aquícola. Estudos realizados por Rocha et al. (2018) abordaram o impacto do parasitismo por Dolops geayi nos parâmetros hematológicos dos tambaquis na região amazônica, região onde esta espécie é muito produzida e consumida. Este estudo verificou que a parasitose pode levar a um quadro de anemia hipocrômica e trombocitopenia, o que compromete a sanidade desta espécie.

O estudo das características sanguíneas pode fornecer subsídios importantes para o diagnóstico das condições mórbidas em populações de peixes (naturais e confinadas) e desta forma, contribuir para a compreensão da fisiologia comparativa, relação filogenética, condições alimentares e outros parâmetros ecológicos (TAVARES-DIAS; MATAQUEIRO, 2004).

É possível observar na figura 3 , que os estudos sobre o efeito da nutrição nos parâmetros hematológicos são frequentes, pois estes atingem a saúde e o bemestar dos peixes. Estudos com relação a suplementação da dieta alimentar em tambaquis foram realizados por Paixão et al. (2017) e Aride et al. (2018), e sobre a 
dieta alimentar em tilápia-do-nilo por Santo et al. (2016) e Sanchez et al. (2017) sendo importantes fontes de informação em relação aos estudos hematológicos e alimentares nessas espécies.

FIGURA 3. Frequência (\%) de objetivos nos estudos sobre hematologia das espécies de peixes no estudo.

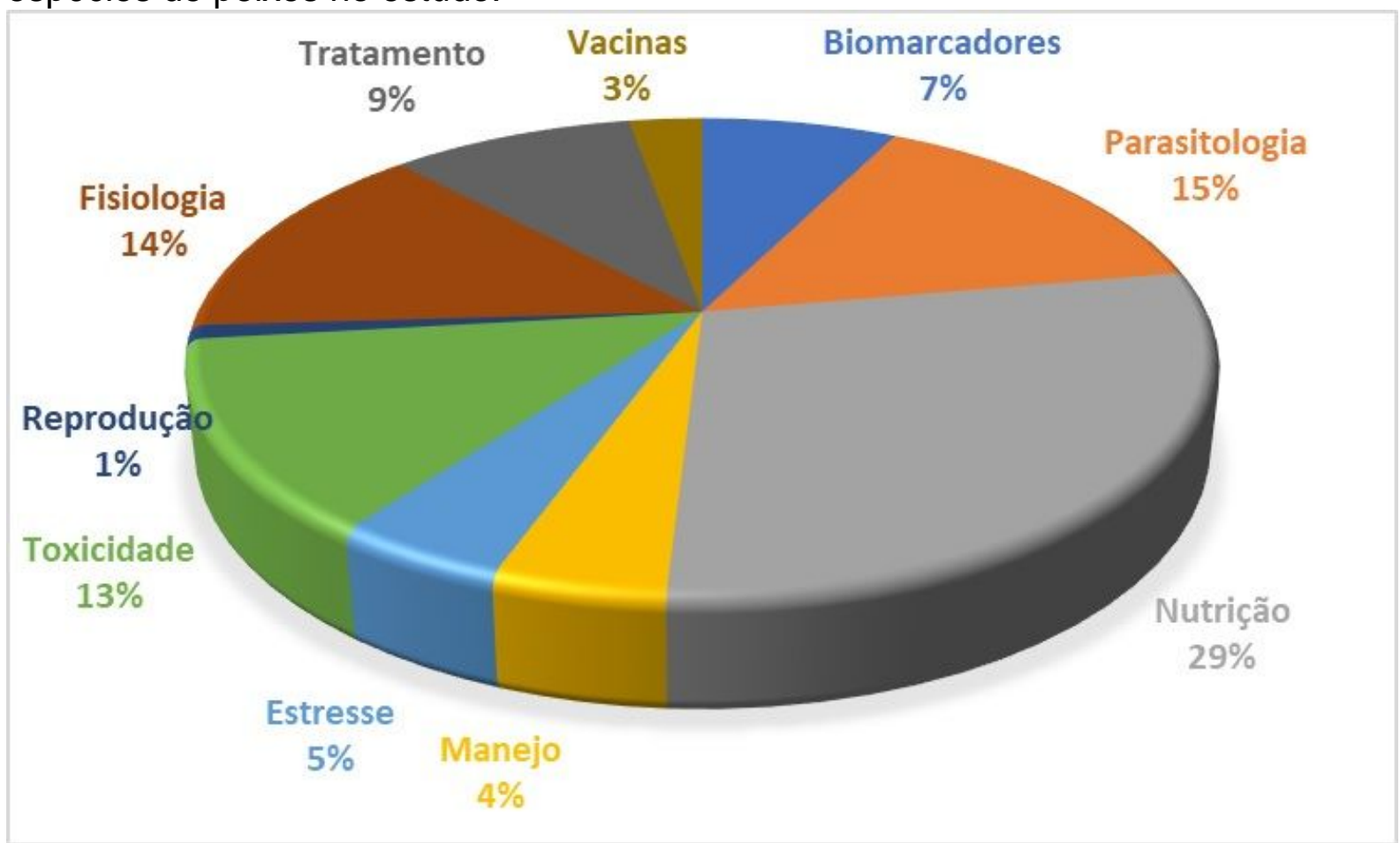

Fonte: Os autores (2020).

No estudo com as tilápias-do-nilo, Araujo et al. (2015), avaliaram os efeitos da adição de lipídeos de óleo de soja, óleo de peixe e sebo bovino e as misturas nas rações sobre a hematologia da tilápia-do-nilo (O. niloticus) antes e após estímulo pelo frio. Jatobá et al. (2018) estudaram possíveis alterações dos parâmetros hematológicos e dos índices zootécnicos na microbiota do trato intestinal em lambaris-do-rabo-amarelo (Astyanax bimaculatus) alimentados com probiótico (Lactobacillus spp.). Este estudo comprovou a eficiência dos probióticos na alimentação dos lambarís, o que pode reverter em ganhos econômicos e aumento no bem-estar animal. A eficácia do uso de probióticos em peixes também foi verificada por Paixão et al. (2017) para o tambaqui (Colossoma macropomum).

Estudos com a inserção de vitaminas na dieta em híbridos de sorubin foi objetivo do trabalho realizado por Rodrigues et al. (2018). Os autores observaram que a inclusão de ácido ascórbico na dieta aumenta a integridade da mucosa intestinal e estimulam a eritropoiese nestes peixes, demonstrando o quanto é importante uma dieta balanceada para a sanidade destes organismos.

O conhecimento acerca das relações entre o efeito do parasitismo e os parâmetros hematológicos é um fator relevante (Figura 3), já que na aquicultura, o parasitismo é uma importante causa de mortalidade em grandes populações de peixes, o que significa em perdas econômicas preponderantes, pois pode causar diminuição no crescimento e no ganho de peso dos peixes cultivados. Assim, é necessário o monitoramento constante do estado de saúde dos peixes em cativeiro. 
Bernardino et al. (2016) observaram que a presença de ectoparasitas promoveram alterações hematológicas e histopatológicas nas brânquias demonstrando que a atividade parasitária nos hospedeiros se apresentou debilitante, pois espécimes com as maiores taxas de infestação apresentaram os menores índices biométricos. Além disso, este estudo mostrou que a simples presença de uma espécie de parasita influencia na infestação da outra espécie hospedeira.

A intensificação nos sistemas de produção de organismos aquáticos tem revelado obstáculos que afetam diretamente a produtividade e o crescimento da atividade, estando a área de Sanidade como um dos principais entraves e que proporciona a base para o estudo e desenvolvimento de novas tecnologias. Os danos causados aos peixes hospedeiros estão relacionados ao parasitismo, bem como à sua forma de alimentação, ao órgão do hospedeiro afetado, às taxas de infecção e ao estado geral do organismo da espécie hospedeira (TAVARES-DIAS, 2006; TAVARES-DIAS et al., 2009).

Em seu estudo, Corrêa et al. (2013) relacionaram os parâmetros hematológicos em alevinos de $H$. malabaricus, demonstrando que mesmo com elevadas intensidades por monogenéticos, não foram observados danos relevantes à saúde do hospedeiro, isto porque os espécimes apresentavam boa condição. Outros estudos sobre o efeito do parasitismo nos padrões normais sanguíneos foi observado por Takemoto et al. (2000) e Rocha et al. (2018), entre outros.

Estudos com relação a toxicidade também se mostraram frequentes (15\%), sendo objeto de diversos estudos científicos. Oliveira Ribeiro et al. (2006) submeteram traíras a dosagens subletais de metais pesados como metil mercúrio por meio da alimentação, mostrando variações nos índices hematológicos e sanguíneos. Neste estudo foi observado aumento dos leucócitos em peixes submetidos ao metilmercúrio.

Mudanças hematológicas e de citogenotoxicidade provocadas por superexposição ao mercúrio e selênio também foram realizadas por Seriani et al. (2015) em tilápias (O. niloticus). Este estudo revelou que o contato com esses contaminantes, são suficientes para provocar alterações nos parâmetros hematológicos do peixe, mostrando portanto, que esta ferramenta além, da influência dos parasitos (LIZAMA et al., 2013), pode ser útil para verificar alterações do ambiente e a relação do ambiente com a saúde dos organismos aquáticos.

Estudo avaliando os parâmetros hematológicos em larvas e juvenis de robalo, Centropomus undecimalis, em decorrência da exposição a diversas concentrações de amônia foi realizado por Pedrotti et al. (2018). Este estudo demonstrou que a toxicidade do ambiente por amônia afeta mais os juvenis que as larvas, e portanto, está relacionada com a idade do animal. Este estudo mostrou também que alterações hematológicas podem servir para avaliar as condições ambientais.

Indicadores de estresse fisiológico, como alguns parâmetros hematológicos e sanguíneos, podem ser úteis para avaliar efeitos de contaminantes, como metais pesados em peixes, mas a aplicação destes achados à elaboração de diagnósticos ambientais necessita de uma investigação mais detalhada e deve ser validado in situ antes de estabelecê-los como biomarcadores.

Estudos hematológicos podem verificar a imunocompetência em peixes, servindo como importante ferramenta para auxiliar na saúde dos animais (PEREIRA et al., 2016). Na busca por otimizar uma metodologia, os estudos de Levy-Pereira et al. (2020) possibilitaram avaliar a fagocitose in vivo em Astyanax altiparanae utilizando Saccharomyces cerevisiae, avaliando a sua resposta hematológica. 
Metodologias como esta, tem a função de propagar tecnologia e consequentemente, conservação da aquicultura.

Diversas técnicas que existem para medir os parâmetros hematológicos advém de adaptações de metodologias tradicionais (RANZANI-PAIVA et al., 2013). Algumas técnicas alternativas vêm sendo estudadas na tentativa de auxiliar nas análises hematológicas em peixes, visto que sua fisiologia se distingue muito. Os biomarcadores como os parâmetros hematológicos permitem detecção precoce das alterações no ambiente e também no peixe, sendo que é possível realizar bioensaios ou análises de cito e genotoxicidade para avaliar estas alterações a longo prazo (VENTURA et al., 2015).

Outros estudos que utilizaram os peixes como marcadores biológicos do impacto no ambiente, também foram observados (Figura 3). Estudo como o de Romão et al. (2006), registrou valores hematológicos significativamente mais baixos em Geophagus brasiliensis e $H$. malabaricus no ambiente rural do que o urbano, sendo considerado desta forma, um ótimo biomarcador.

Estudos relacionando os parâmetros hematológicos e a capacidade de tolerância dos peixes podem ser observados em Silva (2016). Este estudo com Hoplias malabaricus na Represa de Jurumirim-SP mostrou que a espécie é resiliente e tolerante a ambientes impactados. O estresse térmico estudado por Zafalon-Silva et al. (2017) em peixes antárticos também contribuiu para o melhor entendimento quanto à capacidade de tolerância dos peixes em curto e longo prazo.

Por sua vez, Oliveira e Val (2017) estudaram a progressão das mudanças climáticas previstas pelo Intergovernmental Panel on Climate Change for 2100, sobre o crescimento e a fisiologia do tambaqui (Colossoma macropomum) apontando que a espécie consegue se adaptar artificialmente frente às mudanças do ambiente, recuperando os parâmetros sanguíneos normais ao longo do tempo. Este estudo sugere que em ambiente natural, a espécie pode causar desequilíbrios ecológicos na teia alimentar a que pertencem, bem como na ocorrência das mesmas no sistema.

Os índices hematológicos podem servir de importante ferramenta para avaliar as condições do ambiente. No estudo realizado por Silva (2018) com $O$. nilóticus em uma lagoa urbana contaminada, foi observado que em ambientes poluídos, há alteração dos parâmetros, mostrando que os marcadores biológicos devem ser utilizados para o biomonitoramento em ambientes que estão sujeitos à contaminação ambiental.

Estudos hematológicos de peixes são úteis no diagnóstico de muitas doenças, bem como para verificar a extensão dos danos que ocorrem. Esses parâmetros são importantes ferramentas que avaliam o estado de saúde, alterações fisiológicas e patológicas nos peixes e outros animais, atuando como biomarcadores na toxicologia ambiental, deficiências e estresse crônico em sistemas naturais, bem como aquicultura e verificação da qualidade da água. A idade, o tamanho do animal, a maturidade gonadal, as condições de saúde, alimentação, densidade, o ambiente e a própria espécie de peixe promove variações dos parâmetros durante a vida dos peixes (IMTIAZ, et al., 2020). Por isso, devem ser exaustivamente estudados.

\section{CONCLUSÕES}

Com este trabalho pode-se concluir que, o crescimento no setor aquícola promoveu um incremento nos estudos com os parâmetros hematológicos em peixes, principalmente porque a aquicultura está diretamente relacionada com o bem-estar e a sanidade destes animais. Os estudos relacionados a nutrição e suplementação 
animal são os mais frequentes. Já estudos que tratam dos índices sanguíneos com o parasitismo e com o biomonitoramento, são cada vez mais abordados. O uso destes biomarcadores para se avaliar e monitorar o ambiente, bem como a sanidade se tornaram uma ferramenta importante, pois isto afeta não somente a saúde do peixe mas, a saúde humana já que estes organismos são fonte de recurso alimentar.

\section{REFERÊNCIAS}

ARAUJO, C. A.; TEIXEIRA, C.; PEZZATO E. L.; BARROS, M. M. Perfil hematológico de tilápias-do-nilo alimentadas com dietas contendo diferentes lipídeos e estimuladas por baixa temperatura. Universidade Federal Rural do Semi-Árido Mossoró, Brasil. Revista Caatinga, v. 28, n. 1, p 220-227, 2015. Disponível em: https://periodicos.ufersa.edu.br/index.php/caatinga/article/view/3632. Acesso em: 06/2020.

ARIDE, P. H. R., OliVEIRA, A. M., BATISTA, R. B., FERREIRA, M. S., PANTOJALIMA, J. et al. Changes on physiological parameters of tambaqui (Colossoma macropomum) fed with diets supplemented with Amazonian fruit Camu camu (Myrciaria dubia). Brazilian Journal of Biology, v.78, p. 2, p.360-367, May/Aug 2018. Disponível em: <http://dx.doi.org/10.1590/1519-6984.169442>. doi: 10.1590/1519-6984.169442.

ASSOCIAÇÃO BRASILEIRA DE PISCICULTURA. Anuário 2020. Peixe BR da Piscicultura. . São Paulo, 2020. Disponível em: https://www.peixebr.com.br/anuario2020/

BERNARDINO, M. G. S.; SILVA, E. G.; BEZERRA, T. I. C.; LUCENA, R. B.; SATAKE, F. Avaliação ectoparasitológica, hematológica e histopatológica de traíras Hoplias malabaricus Bloch, 1794 provenientes de açudes localizados no município de Sumé, estado da Paraíba. Pesquisa Veterinária Brasileira, v.36, n.7, p. 581586, 2016. Disponível em: <http://dx.doi.org/10.1590/S0100-736X2016000700003> doi: 10.1590/S0100-736X2016000700003

CONTE, F. Stress and the welfare of culture fish. Applied Animal Behaviour Science, v.86, n. 3-4, p. 205-223, 2004. Disponível em: <http://dx.doi.org/10.1016/j.applanim.2004.02.003>.

doi:10.1016/j.applanim.2004.02.003

CORRÊA, L. L.; KARLING, L. C.; TAKEMOTO, R. M.; CECCARELLI, P. S.; UETA, M. T. Hematological parameters of Hoplias malabaricus (Characiformes: Erythrinidae) parasitized by Monogenea in lagoons in Pirassununga, Brazil. Revista Brasileira de Parasitologia Veterinária, v. 22, n. 4, p. 457-462, 2013. Disponível em: <http://dx.doi.org/10.1590/S1984-29612013000400003> doi: 10.1590/S198429612013000400003

EIRAS, A. C. D.; PAVANELLI, G. C.; SILVA-SOUZA, A. T.; TAKEMOTO, R. M.; RANZANI-PAIVA. M. J. T. Avaliação hematológica de espécies de peixe de valor comercial da planície de inundação do rio Paraná, Brasil . Acta Scientiarum: Biological Sciences, v. 22, p. 507-513, 2000. Disponível em: <http://dx.doi.org/10.4025/actascibiolsci.v22i0.2939>.

doi:10.4025/actascibiolsci.v22i0.2939 
IMTIAZ A.; RESHI, Q. M.; FAZIO, F. The influence of the endogenous and exogenous factors on hematological parameters in different fish species: a review. Aquaculture International, v. 28, p. 869-899, 2020. Disponível em: <https://doi.org/10.1007/s10499-019-00501-3>. doi: 10.1007/s10499-019-00501-3

LIZAMA, M. A. P.; FERNANDES, E. S.; ODA, F. H.; MOREIRA, L. H. A.; RIBEIRO, T. S. Parasitos como bioindicadores. In: PAVANELLI, G. C.; TAKEMOTO, R. M.; EIRAS, J.C. (Org.). Parasitologia de peixes de água doce do Brasil. Maringá: Eduem, p. 115-134, 2013.

JATOBÁ, A.; MORAES, K. N.; RODRIGUES, E. F.; VIEIRA, L. M.; PEREIRA, M. O. Frequency in the supply of Lactobacillus influence its probiotic effect for yellow tail lambari. Ciência Rural, v.48, n.10, e20180042, 2018. Disponível em: <https://doi.org/10.1590/0103-8478cr20180042>. doi:10.1590/0103-8478cr20180042

MARTINEZ, C. B. R.; CÓLUS, I. M. S. Biomarcadores em peixes neotropicais para o monitoramento da poluição aquática na bacia do rio Tibagi. In: MEDRI, M. E. et al. Editores. Londrina, PR : M. E. Medri, p. 551-577, 2002. Disponível em: $<$ http://dx.doi.org/10.7213/cienciaanimal.v8i4.11018>.

doi:10.7213/cienciaanimal.v8i4.11018

OLIVEIRA, A.M., VAL, A.L. Effects of climate scenarios on the growth and physiology of the Amazonian fish tambaqui (Colossoma macropomum) (Characiformes: Serrasalmidae). Hydrobiologia 789, 167-178, 2017. Disponível em: <https://doi.org/10.1007/s10750-016-2926-0>. doi: 10.1007/s10750-016-2926-0

OLIVEIRA RIBEIRO, C. A., FILIPAK NETO, F., MELA, M., SILVA, P. H., RANDI, M. A. F. et al. Hematological findings in neotropical fish Hoplias malabaricus exposed to subchronic and dietary doses of methylmercury, inorganic lead, and tributyltin chloride. Environmental Research, v.101, p. 74-80, 2006. Disponível em: <https://doi.org/10.1016/j.envres.2005.11.005>. doi:10.1016/j.envres.2005.11.005

PAIXÃO, A. E. M., SANTOS, J. C., PINTO, M. S., PEREIRA, D. S. P., RAMOS, C. E. C. O. et al. Effect of commercial probiotics (Bacillus subtilis and Saccharomyces cerevisiae) on growth performance, body composition, hematology parameters, and disease resistance against Streptococcus agalactiae in tambaqui (Colossoma macropomum). Aquaculture International 25, 2035-2045, 2017. Disponível em: <https://doi.org/10.1007/s10499-017-0173-7>. doi: 10.1007/s10499-017-0173-7

PEDROTTI, F., MARTINS, M. L., BALOI, M., MAGNOTTI, C., SCHEUER, F. et al. Mortality, hematology, and histopathology of common snook Centropomus undecimalis (Perciformes: Centropomidae) exposed to acute toxicity of ammonia. Journal of Applied Aquaculture, v. 30, n.3, p. 272-284, 2018. Disponível em: <https://doi.org/10.1080/10454438.2018.1443049>.

doi: 10.1080/10454438.2018.1443049

PEREIRA, S. L. A., CHAGAS, E. C., MACIEL, P. O., BENAVIDES, M. V., MAJOLO, C. et al. Agentes patogênicos de tambaquis cultivados, com destaque para registros do rio Preto da Eva, AM. Documentos 127. Manaus: EMBRAPA, 2016. $80 \mathrm{p}$. 
LEVY-PEREIRA, N., YASUI, G. S., EVANGELISTA, M. M., NASCIMENTO, N. F., SANTOS, M. P. et al. In vivo phagocytosis and hematology in Astyanax altiparanae, a potential model for surrogate technology. Brazilian Journal of Biology, v. 80, no. 2, p.336-344, 2020. Disponível em: <https://doi.org/10.1590/1519-6984.205893>. doi: $10.1590 / 1519-6984.205893$

RANZANI-PAIVA, M. J. T.; PÁDUA, S. B.; TAVARES-DIAS, M.; EGAMI M. I. Métodos para análise hematológica em peixes. Maringá: EDUEM, 2013.135p.

ROCHA, M. J. S., JERÔNIMO, G. T., COSTA, O. T. F., MALTA, J. C. O., MARTINS, M. L. et al. Alterações nos parâmetros hematológicos e bioquímicos de tambaqui (Colossoma macropomum) parasitado por espécies de metazoários. Revista Brasileira de Parasitologia Veteterinária, v. 27, n. 4, p. 488-494, 2018. Disponível em: $\quad$ https://doi.org/10.1590/s1984-296120180073>. doi:10.1590/s1984296120180073

RODRIGUES, R. A., DA SILVA NUNES, C., FANTINI, L. E., KASAI, R. Y. D., OLIVEIRA, C. A. L. et al. Dietary ascorbic acid influences the intestinal morphology and hematology of hybrid sorubim catfish (Pseudoplatystoma reticulatum $\times P$. corruscans). Aquaculture International, v. 26, p. 1-11, 2018. Disponível em: <http://dx.doi.org/10.1007/s10499-017-0188-0>. doi: 10.1007/s10499-017-0188-0

ROMÃO, S.; DONATTI, L.; FREITAS, M. O.; TEIXEIRA, J.; KUSMA, J. Blood Parameter Analysis and Morphological Alterations as Biomarkers on the Health of Hoplias malabaricus and Geophagus brasiliensis. Brazilian Archives on Biology and Technology, v.49, n. 3, p. 441-448, 2006. Disponível em: <https://doi.org/10.1590/S1516-89132006000400012>. doi: 10.1590/S151689132006000400012

SANCHEZ, M. S. S., PESSINI, J. E., MORO, E. B., RODRIGUES, M. L., BOSCOLO, W. R. et al. Complexo mineral e vitamínico em dietas para alevinos de tilápia-do-nilo. Boletim de Indústria Animal, 01 December 2017, Vol.74(3), pp.148-155. 2017. Disponível em:

DOI:10.17523/bia.v74n3p148 <https://doi.org/10.17523/bia.v74n3p148>

SANTO, E.F.E., GRASSI, T.L.M., MARCOS, M.T.S., OLIVEIRA, D.L., CAVAZZAN, J.F., et al. Desempenho, sanidade animal e qualidade de filés de tilápias alimentadas com ração suplementada com biomassa bacteriana. Arquivo Brasileiro de Medicina Veterinária e Zootecnia. vol.68, n.2, p.525-534, 2016. Disponível em: <https://doi.org/10.1590/1678-4162-7979>. doi: 10.1590/1678-4162-7979

SCHALCH, S. H. C., ABIMORAD, E. G., ONAKA, E. M., FONSECA, F. S., GARCIA, F. et al. Parâmetros hematológicos de tilápias (Oreochromis niloticus) alimentadas com dieta suplementada de cogumelo Agaricus blazei. Bioikos, v. 29, n.1, p.29, 2015. Disponível em: https://pesquisa.bvsalud.org/portal/resource/pt/vti-379212 ID: vti-379212

SERIANI, R.; FRANÇA, J.; LOMBARDI, J.; BRITO, J.; RANZANI-PAIVA, M. J. Hematological changes and cytogenotoxicity in the tilapia Oreochromis niloticus caused by sub-chronic exposures to mercury and selenium. Fish Physiology and 
Biochemistry, v.41, n.1, p.311-322, 2015. Disponível em: <https://doi.org/10.1007/s10695-014-9984-x> doi: 10.1007/s10695-014-9984-x

SERIANI, R.; RANZANI-PAIVA, M. J. T. Alterações hematológicas em peixes: Aspectos fisiopatológicos e aplicações em ecotoxicologia aquática In: SILVASOUZA, A.T.; PEREZ LIZAMA, M. A.; TAKEMTO, R. M. (Org). Patologia e sanidade de organismos aquáticos. Maringá: ABRAPOA, p.221-242, 2012.

SILVA, E. B. Mucociliary transport, differential white blood cells, and cytogenotoxicity in peripheral erythrocytes in fish from a polluted urban pond. Environmental Science and Pollution Research International, v. 25, n. 3, p.26832690, 2018. Disponível em: <https://doi.org/10.1007/s11356-017-0729-0>. doi: 10.1007/s11356-017-0729-0

SILVA, R. J. (Org.). Integridade ambiental da represa de Jurumirim: ictiofauna e relações ecológicas [online]. São Paulo: Editora UNESP, 2016, 249 p. ISBN 978-856833-478-2. Disponível em: <https://doi.org/10.7476/9788568334782>. doi: $10.7476 / 9788568334782$.

TACHIBANA, L., DIAS, D. C., ISHIKAWA, C. M., GONÇALVES, G. S., NATORI, M. M. et al. Recentes avanços dos estudos e utilizações de probióticos na piscicultura. In: RANZANI-PAIVA, M. J.; TAKEMOTO, R. M.; LIZAMA, M. A. P.; PERAZZOLO, L. M.; ROSA, R. D. (Org.). Biotecnologia e sanidade de organismos aquáticos, $p$. 42-80. 2019.

TAKEMOTO, R. M.; SILVA-SOUZA, A. T.; PAVANELLI, G. C.; RANZANI-PAIVA, M. J. T. Características hematológicas e fator de condição relativo $(\mathrm{Kn})$ associados ao parasitismo em Schizodon borellii (Osteichthyes, Anostomidae) e Prochilodus lineatus (Osteichthyes, Prochilodontidae) do rio Paraná, região de Porto Rico. Acta Scientiarum : Biological Sciences, v. 22, p. 515-521, 2000. Disponível em: <https://doi.org/10.4025/actascibiolsci.v22i0.2940>. 10.4025/actascibiolsci.v22i0.2940

TAVARES-DIAS, M., ISHIKAWA, M. M., MARTINS, M. L., SATAKE, F., HISANO, H. et al. Hematologia: ferramenta para o monitoramento do estado de saúde de peixes em cultivo. In: Tópicos Especiais em Saúde e Criação Animal. SARAN-NETO; M.; POZZOBON-SORIA, W. S. (Orgs.). 1ํㅡㄹ ed. São Carlos: Pedro \& João Editores, 2009.

TAVARES-DIAS, M.; MATAQUEIRO, M. I. Características hematológicas, bioquímicas e biométricas de Piaractus mesopotamicus Holmberg, 1887 (Osteichthyes: Characidae) oriundos de cultivo intensivo. Acta Scientiarum Biological Science. Maringá. v. 26, n. 2, p. 157-162, 2004. Disponível em: <http://hdl.handle.net/11449/67700 >. Acesso em: 03/12/2020.

TAVARES-DIAS M. A morphological and cytochemical study of erythrocytes, thrombocytes and leukocytes in four freshwater teleosts. Journal of Fish Biology, v.68, p.1822-1833, 2006. Disponível em: <https://doi.org/10.1111/j.10958649.2006.01089.x>. doi: 10.1111/j.1095-8649.2006.01089.x 
UEDA I. K.; EGAMI, M. I.; SASSP, W. DA S.; MATUSHIMA, E. R. Estudos hematológicos em Oreochromis niloticus (Linnaeus, 1758) (Cichlidae, Teleostei) Parte I. Brazilian Journal of Veterinary Research and Animal Science, v. 5, p. 270-275, 1997.

VENTURA, A. S.; CORSINI, F. E.; ARAÚJO, G. A. M. Hematologia como biomarcador de contaminação ambiental em peixes. Sangue, poluentes, metais pesados. Revista Nutri-time, v. 12, n. 6, 2015. Disponível em: <http://www.nutritime.com.br/arquivos internos/artigos/354 - 4500-4507_-

NRE_12-6_nov-dez_2015.pdf>. Acesso em: 12 out. 2020.

ZAFALON-SILVA, B., ZEBRAL, Y. D., BIANCHINI, A., ROSA, C. E., MARINS, L. F. et al. Erythrocyte nuclear abnormalities and leukocyte profile in the Antarctic fish Notothenia coriiceps after exposure to short- and long-term heat stress. Polar Biology 40, p.1755-1760, 2017. Disponível em: <https://doi.org/10.1007/s00300017-2099-y>. doi: 10.1007/s00300-017-2099-y 